\title{
Membrane Guided Regeneration in Periodontal Tissues
}

\author{
Jaisika Rajpal ${ }^{1,},{\text { Aakash } \text { Arora }^{2}, \text { Ruchika Prasad }^{3} \text { and Madhav Mukund Gupta }}^{4}$ \\ ${ }^{1}$ Dept. of Periodontology, Subharti Dental College, Meerut, India \\ ${ }^{2}$ Dept. of Oral and Maxillofacial Surgery, Subharti Dental College, Meerut, India \\ ${ }^{3}$ Dept. of Oral Medicine, Diagnosis and Radiology, Sardar Patel Post Graduate Institute of Dental \& Medical \\ Sciences, Lucknow, India \\ ${ }^{4}$ Dept. of Periodontology, Faculty of Dental Sciences, CSMMU, Lucknow, India
}

\begin{abstract}
Periodontal regeneration is the restoration of lost periodontium or supporting tissues and includes the formation of new alveolar bone, new cementum and new periodontal ligament. The concept of GTR is based on the exclusion of gingival connective tissue cells and prevention of epithelial down growth into the wound, thereby allowing cells with regenerative potential (PDL and bone cells) to enter the wound first. GTR consists of placing barriers of different types to cover the bone and periodontal ligament thus temporarily separating them from gingival epithelium. Excluding the epithelium and gingival connective tissue from the root surface during the post-surgical healing phase not only prevent epithelial migration into the wound but also favors repopulation of the area by cells from the periodontal ligament and bone. Purpose and Scope -This review discusses the rationale for using guided tissue regeneration therapy. The review not only attempts to clarify the concept of selective tissue regeneration using non-resorbable and resorbable barriers, but to discuss differences in healing events after treatment with the two types of barriers together with their significance in periodontal therapy. At present, barrier membranes have potential clinical use in promoting periodontal tissue regeneration if patients to be so treated are selected appropriately. Research is still necessary to determine the critical period for guiding the ingrowth of new attachment forming cells and also to further clarify the concept of GTR involving the "wrong cell type" which inhibits periodontal tissue regeneration.
\end{abstract}

Keywords: Guided tissue regeneration, epithelial migration, cell exclusion, barrier membranes.

\section{INTRODUCTION}

The ultimate goal of periodontal therapy is predictably regeneration of a functional attachment apparatus destroyed by periodontitis. Regeneration should be distinguished from repair. Regeneration is defined as the type of healing which completely replicates the original architecture and function of a part. It involves the formation of a new cementum, periodontal ligament, and alveolar bone. Repair, on the other hand, is merely a replacement of loss apparatus with scar tissue which does not completely restore the architecture or the function of the part replaced. The end product of repair is the establishment of long junctional epithelium attachment at the tooth-tissue interface. Traditional therapeutic modalities usually failed to predictably regenerate the periodontal tissue lost due to disease process.

\section{PRINCIPLE OF GUIDED TISSUE REGENERATION}

The principle of guided tissue regeneration (GTR) can be applied and may result in re-constituion of the functional periodontal apparatus (new cementum, periodontal ligament, and alveolar bone). Procedures

*Address correspondence to this author at 45/A Aashirwad Bhawan, Beside Maittri Niwas Guest House, Krishna Nagar, Kanpur Road Lucknow-226023, India; Tel: 0522-2473104; Mob: 09451950222;

E-mail: drjaisikarajpal@gmail.com which relies heavily in the principle of GTR involves those whose end results is the complete regeneration of periodontal structures which were lost due to periodontal disease, those whose objectives is the ridge augmentation to allow proper placement of osseointegrated implant, and also the procedures which are utilized in treatment of fraction and recession defects.

GTR procedures attempt to achieve periodontal regeneration through biologic principles of differential tissue response.

\section{HISTOLOGY OF WOUND HEALING}

To further understand principle of GTR, we must review the histology of wound healing. In the site of periodontal healing, we have four situations which may occur. First, the epithelium will try to migrate from the wound margin down to the base of the sulcus. If this occurred, the reestablishment of the pocket or in the best scenario, long junctional epithelium will be established. Thus for regeneration to occur, epithelial migration must be prevented. Secondly, the connective tissue will try to grow into the area of the defect. If this occurs, the end result will be external resorption at the connective tissue-root interface. Thirdly, if the bone cells are allowed to repopulate the area of defect, ankylosis or resorption will occur at the junction of bone 
to tooth interface [1]. Karring et al. in 1980 demonstrated this in beagle dogs, when the roots were extracted and transplanted into a surgically created alveolar bone in the edentulous part of a jaw, ankylosis and root resorption occur. Finally, the cells of periodontal ligament, if allowed to repopulate the root surface, the regeneration can be established [2]. Nyman et al. in 1982 showed in a study in monkey with the use of millipore filter to exclude the epithelium and the gingival connective tissue. After three months, the histological specimen demonstrated new attachment, new cementum, and new bone. He further confirmed this result with the follow up study on a root surface in human using the principle of GTR [3]. A block biopsy of a lower central incisor at three months after surgery showed new cementum and with inserting collagen fibers extending five millimeter coronally from the apical level of root. Melcher in 1976 reported these four tissue compartments in the periodontium and that each of these tissues was capable of producing a unique cell phenotype [4], and that the type of healing following periodontal therapy depended on the phenotype of the cells which first repopulated the root surface (Figure 1).

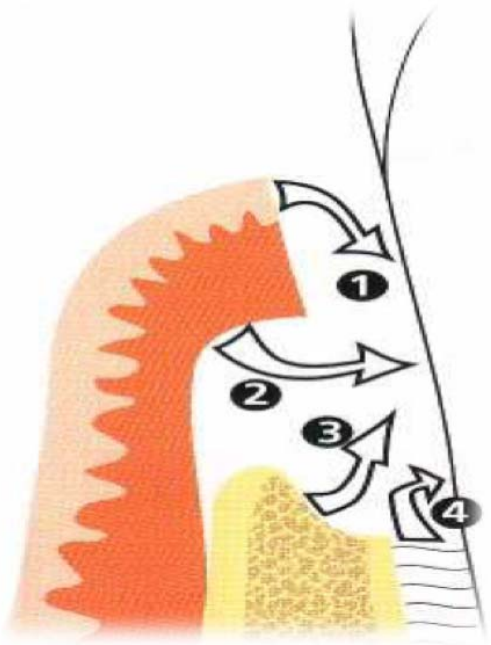

Figure 1: Melcher's Principle- 1. Epithelial cells, 2. Cells derived from the gingival connective tissue, 3 . Cells derived from the bone, 4 . Cells derived from the periodontal ligament.

\section{GUIDED CELL REPOPULATION}

The concept of GTR is based on the exclusion of gingival connective tissue cells and prevention of epithelial down growth into the wound, thereby allowing cells with regenerative potential (PDL and bone cells) to enter the wound first.

This method derives from the classical studies of Nyman, Lindhe, Karrin and Gottlow and is based on the assumption that only the periodontal ligament cells have the potential for the regeneration of the attachment apparatus of the tooth [5].

GTR consists of placing barriers of different types to cover the bone and periodontal ligament thus temporarily separating them from gingival epithelium. Excluding the epithelium and gingival connective tissue from the root surface during the post-surgical healing phase not only prevents epithelial migration into the wound but also favors repopulation of the area by cells from the periodontal ligament and bone.

The ideal properties of a barrier membrane are - (1) biocompatibility, (2) space maintenance, (3) cellocclusiveness, (4) good healing properties, and (5) Resorbability [6].

GTR has proved to be more effective than open flap debridement in the gain of clinical attachment and probing depth reduction in the treatment of intrabony and furcation defect.

\section{BARRIER MEMBRANES}

The principle of GTR thus involves the use of a physiological barrier which is placed over the denuded lesions in such a way that all periodontal tissue except the periodontal ligament cells and the alveolar bone are prevented from contacting with the root. The cells of periodontal ligaments are the only ones which seem to have the capacity to form new attachment. Cells of periodontal ligament migrate and differentiate faster than those of bone, thus even though bone cells were allowed to migrate to the area along with the cells of PDL, we would expect the cells of PDL will repopulate along the root surface [7].

The use of a barrier has first been reported by Younger in the Dental Cosmos of 1904, of which a Japanese paper saturated with liquid celluloid was used to form a protecting wall over the roots and the edge of the gingiva. "The covering hardens and forms a protective shield to the granulation tissue which grows up and fills the space between the roots..." Prichard in 1957 further stated that cells that are necessary for the genesis of periodontal ligament, cementum, and alveolar bone are available in the area that borders the bony deformity. This lead to Melcher's principle in 1976 which classified the four tissue types which will repopulate the root surface (as described previously) [8]. Further investigations in the 1970's and 80's supported Melcher's concept. Caton et al. examined healing following four different modalities of periodontal 
treatment (scaling and root planing, modified widman flap with debridement alone or in combination of autogenous or synthetic bone graft). The end results demonstrated the establishment of long junctional epithelium between the gingival connective tissue and the root surface upon healing. This finding supported other similar studies that conventional nonsurgical and surgical periodontal therapies usually resulted in repair rather than regeneration.

\section{EPITHELIAL EXCLUSION BY MEMBRANE}

The effects of epithelial exclusion were further investigated by Nyman in 1980. When root was allowed to contact alveolar bone, ankylosis and root resorption occurred. When root was allowed to contact the gingival connective tissue and the root surface had been denuded of periodontal fiber, the root resorption occurred. These observations suggested that exclusion of gingival epithelium alone does not promote periodontal regeneration. His further study with the millipore filter in 1982 reported that the periodontal ligament cells has a considerable potential for periodontal regeneration, and that this potential is manifested only when the gingival epithelium and connective tissues are excluded from the periodontal wound. He further followed up on the human study on the selected mandibular incisor. Again, histological evaluation revealed new cementum with inserting collagen fibers extending $7 \mathrm{~mm}$ from the apical level of root planing in a coronal direction.

In 1986, Got low et al. presented a case report of 12 periodontally involved teeth from 10 patients treated using this biologic principle. Eleven of these teeth formed the experimental group and were treated by flap elevation, granulation, tissue debridement, scaling and root planing followed by placement of ePTFE barrier (Gore-Tex membrane). The remaining tooth was also surgically treated but without the placement of barrier as the controls. Clinical results from reentry indicated significant gain in clinical attachment and probing depth reduction, as well as an apparent bone fill in some of the previously presented osseous defects [9]. Histological observations disclosed a substantial amount of periodontal regeneration in all the teeth treated with the barrier. These findings demonstrated that periodontal regeneration could be predictably obtained in humans by placing the physical barrier, which selectively excludes gingival epithelium and connective tissue and favors periodontal ligament repopulation of the root surface.
In 1986, Melcher performed an experiment to identify the source of the cells that invade the area of wound healing. Rat calvarium bone cells were incubated with root slices in the absence of periodontal ligament cells. He noted the synthesis of a cementum like substance on a root slices. Mc Culloch later demonstrated that cell of the periodontal ligament is not a closed compartment, and that paravascular cells in the endosteal spaces of the alveolar bone may contribute to the periodontal ligament cell population [9].

\section{CELLULAR MIGRATION}

The cellular process involved in the development of the periodontium and in wound healing must be understood in order to comprehend the regeneration concept in periodontal defects. The major type of cell in the periodontal ligament is the fibroblast. Fibroblasts are located throughout the connective tissues of the body, where their role is to maintain the extracellular matrix substance. The periodontal fibroblast is capable of extensive protein and collagen synthesis and that it responds well to the molecular mediators during the process of wound healing. Fibroblast apparently has the potential to develop into different type of cells during wound healing, depending on the molecular mediator that stimulates it [10]. The precursor cells of the periodontal ligament, in this case the fibroblast, can differentiate into collagen fibers, osteoblasts, or cementoblasts, depending on their position. Other studies have reported that cell populations are mixed throughout the periodontal ligament. Cell migration in the periodontal ligament seems to occur starting at the bone interface and continuing along the collagen fibers. There must be a mechanism which selectively activates bone precursor cells to repopulate the area and establish a new tissue exactly like the originating tissue, with each type of cell in its proper position. Specific cellular types that repopulate the wound defect will determine the form and type of tissue that will be created. The proliferation of the proper type of cells in their proper position may be regulated via molecular growth factors which are thought to be responsible for specifically stimulating the proliferation of cementum, periodontal ligament, and bone cells [11, 12]. The ultimate goal of GTR is to use a mechanical barrier to provide the environment necessary for the body to utilize its natural healing potential and to regenerate lost and absent tissue. Ultimately, the efficacy of periodontal membranes in conjunction with wound healing is the result of a combination of different mechanisms-mechanical, cellular, and molecular. 
Some examples of these mechanism are the prevention of fibroblast mass action, the prevention of contact inhibition by cell interaction, the exclusion of cell derived soluble inhibitory factors, the local concentration of growth stimulatory factors, and the stimulatory properties of the periodontal membrane itself.

\section{CLINICAL APPLICATIONS OF GTR}

The clinical indication for the use of GTR are the class II furcation defects, two or three walled vertical, interproximal, and circumferential intrabony periodontal defects (Figure 2). Class III furcation may be treated with GTR but with less predictability of success. Other clinical indication of GTR are the ridge augmentation (can also be referred to as guided bone regeneration), and the treatment of gingival recession. Sites which may be at risk for postsurgical recession are best treated with nonresorbable barriers, since barrier exposure may accelerate resorbable barrier degradation. Bone graft may be used in combination with GTR for the supporting purpose to prevent the collapse of the membrane. Success of GTR treatment relies heavily on the ability to stabilize the blood clot. Blood clot stabilization is the major prerequisite for the regeneration to evolve. Wickejolze has shown without the blood clot (with the use of heparin to dissolve the clot on root surface), the regeneration failed to occur. Other factors which aided in successful GTR technique are oral hygiene, adequate initial hygienic therapy, proper flap selection and management, adequate debridement to completely remove all granulation and soft tissue at the treated site, the decortication of the bony defect underneath the membrane to stimulate the formation of a blood clot, adequate adaptation of membrane to prevent epithelium to migrate underneath the membrane, adequate debridement to denude the bone of the defect site, adequate size and shape barrier chosen (extending 2-3mm pass the border of the defect), and finally, complete coverage the membrane underneath the flap upon suturing. Sutures may be removed after 7-10 days. If PTFE sutures are used, they may be allowed to remain for a longer period of time in order to aid in flap adaptation since this type of suture does not cause wicking and trapping of bacteria. If the barrier is nonresorbable, it is removed approximately 4-8 weeks. The most important period of cell migration and proliferation are the first 30 days. If the membrane can be maintained underneath the flap for this initial period, we can achieve closely or maximal amount of regeneration. In short, clot formation and stabilization, space provision, revascularization, epithelial cell exclusion, and complete gingival

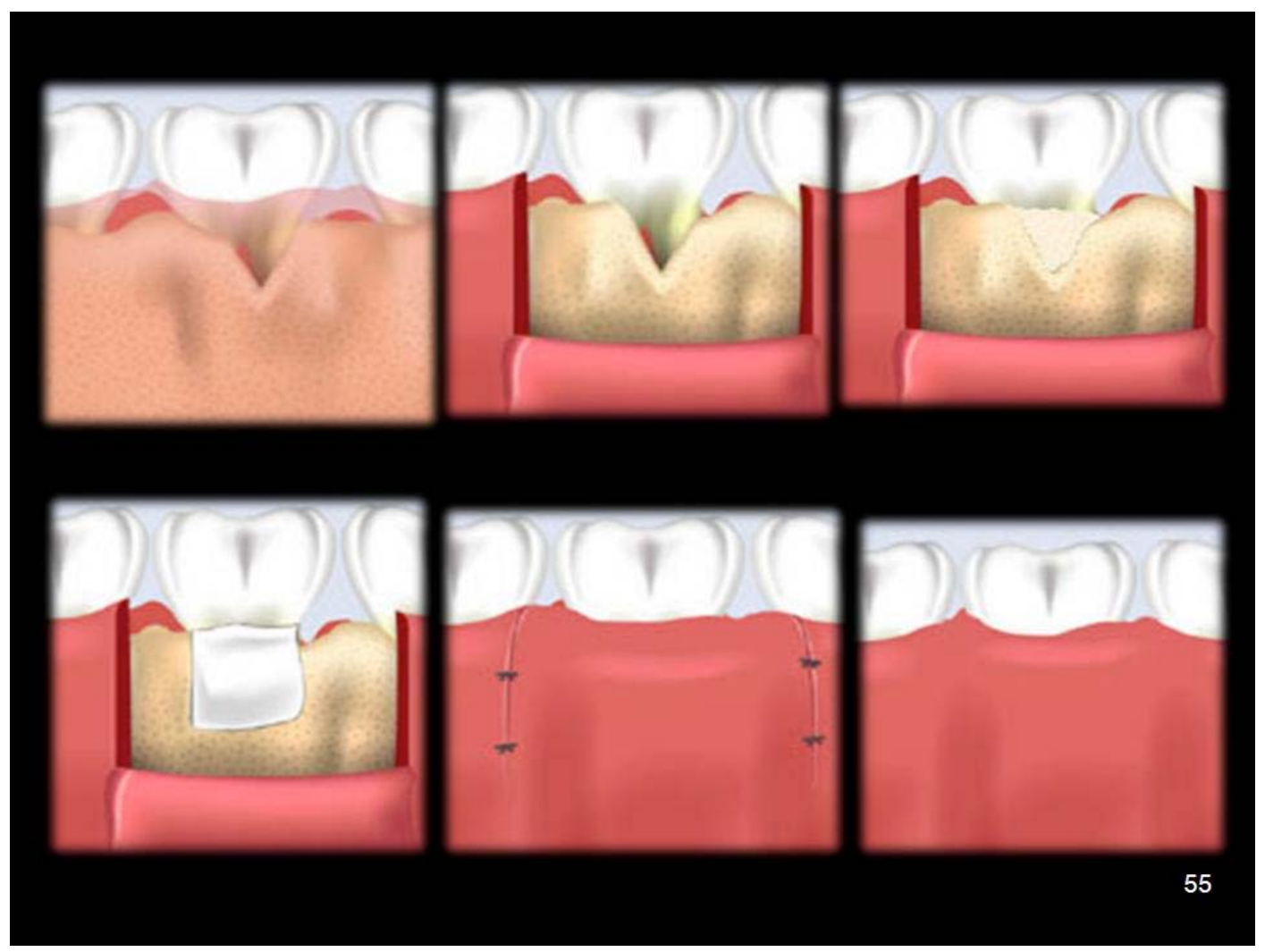

Figure 2: Diagrammatic representation of surgical procedure for placement of GTR for periodontal regeneration. 
coverage are desirable characteristics to achieve in any GTR procedure [13].

\section{TYPES OF BARRIER MEMBRANES}

1. Nonresorbable barrier included the regular and the titanium reinforced Gore-Tex membrane. The basic molecule of PTFE consists of a carboncarbon bond, with four attachments of fluorine atoms to form the polymer. The carbon-fluorine bond is one of the strongest bonds known among organic compounds. The highly electronegative fluorine atoms form a protective sheath over the chain of carbon atoms. This sheath shields the carbon chain from most chemicals, is responsible for the chemical inertness and stability of the polymer, and lowers its surface energy. PTFE is extremely resistant to even the most highly corrosive chemicals. The combination of chemical, thermal, and mechanical properties makes PTFE one of the most inert substances known to man. The expanded PTFE involved a microstructure consisting of solid nodes interconnected by fine, highly oriented fibrils. The expanded PTFE nodes and fibrils provide unique porous structures. The expanded PTFE is neither woven nor knit and thus will not fray nor is abrasive. The microstructure of the material can be adjusted to provide a matrix for cellular attachment and ingrowths. The Gore-Tex membrane took advantage of the biocompatible and porous structure of expanded PTFE. It contains the open microstructure portion (the central portion) which is designed to inhibit or retard the apical migration of epithelium during the early phase of wound healing. This phenomenon is referred to as contact inhibition. The epithelium recognized the tissue attached to the open microstructure as non foreign and stops migration beyond it. This limits the pocket formation on the outer surface of the membrane and thereby enhances the tissue health. When placed subgingivally, the open microstructure helps to stabilize the clot and inhibits or retards rapid migration of the epithelium during early healing. The Gortex periodontal material (GTPM) also consists of the outer partially occlusive membrane which serves as the barrier between the gingival connective tissue and tooth root. This created a protected space over the defect that allows the cells from the remaining periodontal ligaments to selectively repopulate the root surface. It is also designed to allow for incorporation by surrounding tissues and thereby may retard apical migration of epithelium during the later phases of healing. The Gore-Tex suture is provided for use with the GTPM. It is nonabsorbable, monofilament PTFE sutures that have been expanded to produce a porous microstructure. It elicits minimal tissue response and excellent handling. It has non-wicking properties which prevent bacteria and plaque accumulation at the healing site (Figure 3 ).

2. Resorbable barriers have already obtained approval for use in the United States (Guidor, Resolut, Bio-mend, and Attrisorb). They are composed of polylactic/polyglycolic acid and a citric acid ester. The design of Guidor is a

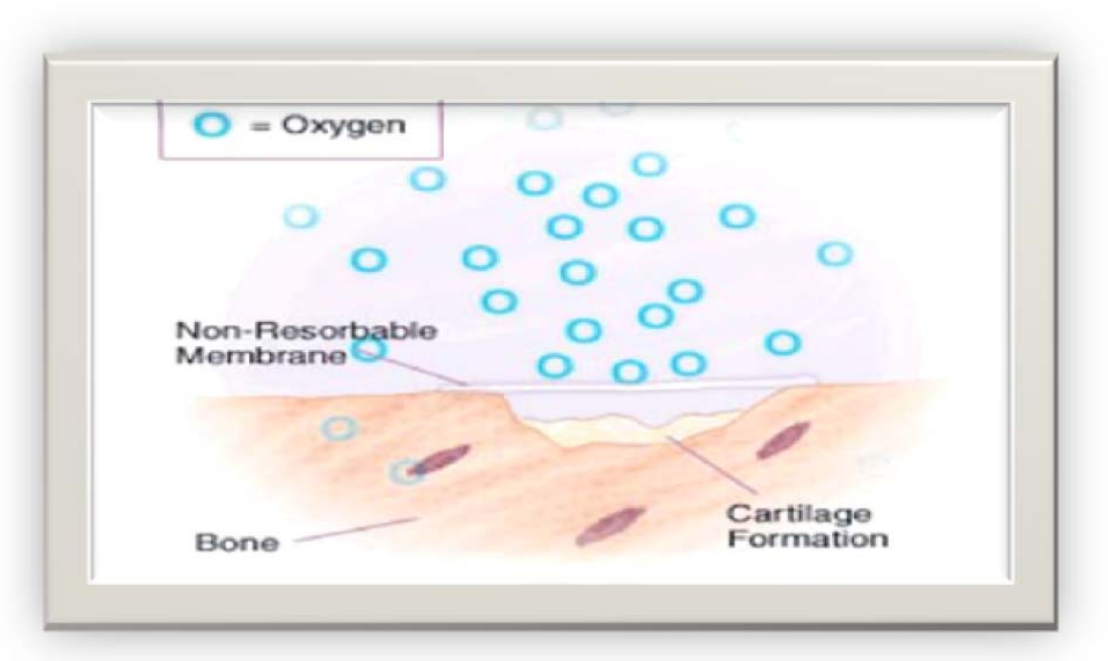

Figure 3: Mechanism of action of non-resorbable membrane. 
multilayer matrix, which facilitates the ingrowth of gingival connective tissue from the inner aspect of the periodontal flap. This ingrowth is assumed to retard and prevent the apical downgrowth of gingival epithelium. Resorbable barrier provided the advantage of eliminating the second surgery to retrieve the undegraded barrier membrane (Figure 4). This second surgery may disrupt initial healing and limit the overall attachment gain. Another benefit of using Guidor is its application in treatment of gingival recession in the field of periodontal plastic surgery. The use of the membrane in single site recession eliminated the problems associated with conventional grafting which includes color and tissue texture alteration and patient discomfort due donor site on the palate. In a survey by Roccuzzo, all patients preferred the Guidor treatment for better comfort. Patient clearly preferred the single site GTR technique since they can avoid the palatal wound. With Guidor, we may anticipate the regeneration of supporting apparatus, whereas with the conventional grafting, long junctional epithelium or repair is expected (Figure 5).

\section{VARIOUS STUDIES DONE FOR EVALUATION OF GTR}

Gotlow et al. evaluated the use of resorbable barrier in recession type and interproximal defects in nonhuman primates. Clinical healing following surgery progressed with minimal or no gingival inflammation [14]. Histological evaluation demonstrated the new cementum with inserting periodontal ligament fibers extending to the coronal border of barrier together with new bone formation. After 6 months, the barrier was completely resorbed. He followed with study of treatment of infrabony defects in monkey with Guidor and Goretex periodontal materials. 2 out of 30 sites were treated with resorbable barrier and 21 out of 30 areas treated with the nonresorbable barrier become exposed following surgery. The amount of new cementum and periodontal ligament regeneration was $72 \%$ and $63 \%$ defect height for the resorbable and nonresorbable respectively, while the corresponding

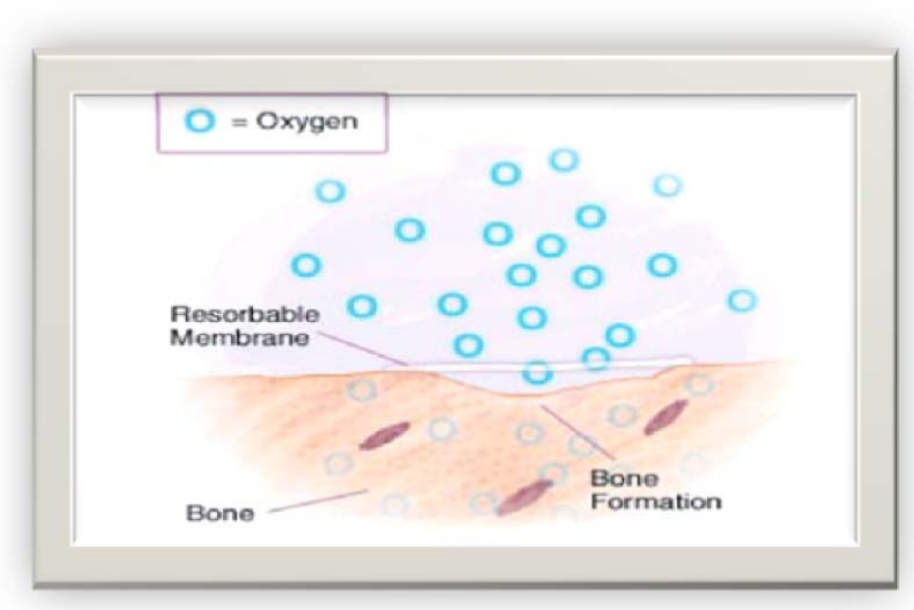

Figure 4: Cell exclusion mechanism of resorbable membranes.

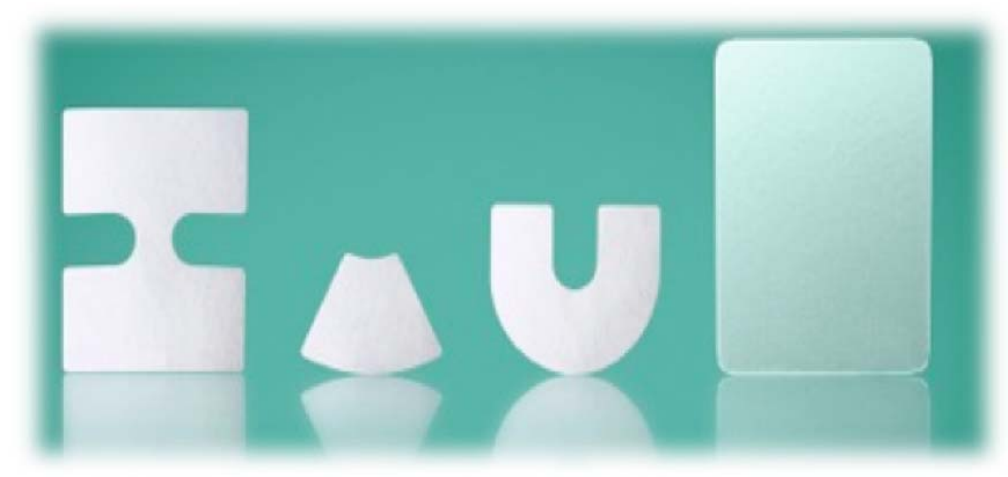

Figure 5: Various shapes of available resorbable membranes. 
percentage for bone regeneration were $89 \%$ and $87 \%$. It was concluded that the difference observed in the healing response of both materials was due to the barrier exposure and to the surgical trauma, occurring during the removal of nonresorbable barriers.

Alan Polson, Steven Garrett, Norman Stoller, Gary Greenstein, Anne Polson, Charles Harrold, and Larry Laster involved in the multicentre study of Guided Tissue Regeneration in human furcation defects after using a biodegradable barrier. 29 patients with class II furcation defects were treated using polylactic acid biodegradable barrier. At twelve month post surgery, there was clinically and statistically significant improvement in mean pocket depth reduction $(2.2 \mathrm{~mm})$ and attachment level vertical gain $(1.7 \mathrm{~mm})$, and attachment level horizontal gain $(2.5 \mathrm{~mm})$. These results indicated favorable clinical regenerative outcomes after using this barrier material in class II furcation defects in humans [15].

Genon et al. presented data from 16 cases in which the Guidor matrix barrier was used in conjunction with the coronally position flap to treat the recession defect. Gingival recession was reduced on average by $3.7 \mathrm{~mm}$ with gingiva up to or within $1 \mathrm{~mm}$ of cemento-enamel junction in 9 of 16 patients. Clinical attachment level improved by a mean of $3.9 \mathrm{~mm}$.

Giampaolo Pini Prato, Carlo Clauser, Cortellini, Carlo Tinti, Giampaolo Vincenzi, and Umberto Pagliaro reported the four year follow up results of a clinical trial of which guided tissue regeneration versus mucogingival surgery were used in the treatment of human buccal recession. The result showed that average reduction in the recession was similar in the two groups while probing depth reduction and clinical attachment level were greater in the GTR group [16].

Biomed is the type I collagen membrane, derived from Bovine Achilles tendon. Since type I collagen is the most predominant protein in human connective tissue including periodontal tissue, it is a natural selection as a resorbable material for the guided tissue regeneration. Due to its ability to promote platelet aggregation, the collagen is known to be a natural haemostatic agent, and this characteristic may facilitate early wound stabilization and maturation and enhancing fibrin linkage and initial blood clot formation. It also is chemotactic for fibroblast in vitro and may serve as biologic scaffold for the ingrowth of endothelial cells, other vascular elements, and progenitor cells from the periodontal ligament. The biomend membrane is biodegradable [17] with average retention time of 6-7 weeks and total absorption documented at 8 weeks. IT demonstrated tear resistance, semipermeable for epithelial cell exclusion but still allowed for nutrient diffusion. Its indication are for class II furcation defects.

\section{FUTURE PERSPECTIVES FOR THE GTR}

One of the main shortcomings of all clinical regenerative procedures is relatively high variation and low predictability of clinical attachment and bone gain $[18,19]$. Since products should retain their biocompatibility, but have better efficacy, this could be accomplished through usage of new techniques developed in similar biomedical branches. The condition for predictable tissue regeneration is stimulation of precursor cells with necessary messenger molecules. Control of progenitor cells in periodontal healing process is complex and mostly unknown. It seems that various local factors play a role in attracting the cells to the wound space from bone marrow and periodontal ligament spaces. A good example for new trends is membrane surface modification, especially incorporation of adhesion molecules which should be able to physiologically stimulate cell and tissue adhesion. The next step could be application of specific adhesion molecules resulting in tissue selection on the membrane surface [20]. There is enough evidence indicating the important role of adhesion molecules in periodontal health and disease. In order to minimize detrimental microbial influence on the regenerative procedure, addition of antimicrobial substances has been investigated. Antimicrobial action might beneficially influence early phases of wound healing and thus improve the outcome of the regenerative procedure. However, one clinical investigation found no advantage for metronidazole as an additive present in the tested resorbable membrane [21]. Addition of growth and differentiation factors has been investigated. There is enough evidence that certain growth factors and cell mediators can act on competent cells in the healing of periodontal wound space and regeneration of tissues such as cementum and bone.

\section{SUMMARY}

The use of GTR membranes can lead to significant periodontal regeneration, and formation of cementum with inserting fibers, although complete regeneration has never been reported. The advantage of resorbable membranes is unnecessary surgical removal, while collagen membranes have additional advantages 
related to biological properties of collagen itself. Products used for GTR should maintain biocompatibility, but develop better efficacy, possibly using new techniques and technologies that have been developed and applied in neighbouring medical branches.

Application of specific adhesion molecules should lead to tissue selection on the membrane surface. Addition of antimicrobial substances might minimize the influence of microbial contamination on regenerative outcome, growth factor incorporation should stimulate regenerative biologic potential of bone and cementum. Combination of these molecules might lead to significant changes in the outcome of GTR procedures. Further investigations are needed to improve clinical outcome, because there is insufficient proof of the clinical efficacy of these concepts. Better understanding of factors influencing regenerative procedure will probably improve predictability of therapy of bone defects around natural teeth and implants.

\section{REFERENCES}

[1] Caffesse RG, Nasjletti CE, Morrison EC, Sanchez R. Guided tissue regeneration: comparison of bioabsorbable and nonbioabsorbable membranes. Histological and histometric study in dogs. J Periodontol 1994; 65: 583. http://dx.doi.org/10.1902/jop.1994.65.6.583

[2] Nyman S, Karring T, Lindhe J, Planten S. Healing following implantation of periodontitis affected roots into the gingival connective tissue. J Clin Periodontol 1980; 7: 394 http://dx.doi.org/10.1111/j.1600-051X.1980.tb02012.x

[3] Nyman S, Gotlow J, Karring T, Lindhe J. The regenerative potential of the periodontal ligament. An experimental study in monkey. J Clin Periodontol 1982; 9: 257. http://dx.doi.org/10.1111/j.1600-051X.1982.tb02065.x

[4] Melcher A. Repair of wound in the periodontium of the rat. Influence of the periodontal ligament on osteogenesis. Arch Oral Biol 1970; 15: 1183.

http://dx.doi.org/10.1016/0003-9969(70)90010-5

[5] Nyman S, Lindhe J, Karring T, Rylander H. New attachment following surgical treatment of human periodontal disease. $J$ Clin Periodontol 1982; 9: 290. http://dx.doi.org/10.1111/j.1600-051X.1982.tb02095.x

[6] Kao RT, Conte G, Nishimine D, Dault S. Tissue engineering for periodontal regeneration. J Calif Dent Assoc 2005; 33: 205-15.

[7] Wang HL, O'Neal R, MacNeil L. Regenerative treatment of periodontal defects utilizing a bioresorbable collagen membrane. Practical Periodontics Aesthetic Dentistry 1995; 7: 59.

[8] Melcher A, Cheong T, Cox J. Synthesis of cementum like tissue in vitro by cells cultured from bone. A light and electron microscope study. J Periodontal Res 1986; 21: 592. http://dx.doi.org/10.1111/j.1600-0765.1986.tb01497.x

[9] Gotlow J, Laurell L, Lundgren D, Mathisen T, Nyman S, Rylander $\mathrm{H}$, Bogentoft $\mathrm{C}$. Periodontal tissue response to a new bioresorbable guided tissue regeneration device: a longitudinal study in monkeys. Int J Periodontics Restorative Dentistry 1994; 14: 437.

[10] Gotlow J, Nyman S, Karring T, Lindhe J. New attachment formation as the result of controlled tissue regeneration. $J$ Clin Periodontol 1984; 11: 494.

http://dx.doi.org/10.1111/j.1600-051X.1984.tb00901.x

[11] Lindhe S, Polson A, Zander H. Relationship between periodontal injury, selective cell repopulation and ankylosis. J Periodontol 1974; 45: 725. http://dx.doi.org/10.1902/jop.1974.45.10.725

[12] Stahl S, Froum S. Histologic healing response in human vertical lesions following the use of osseous allografts and barrier membranes. J Clin Periodontol 1991; 18: 149. http://dx.doi.org/10.1111/j.1600-051X.1991.tb01705.x

[13] Caranza N. Textbook of Clinical Periodontology. $8^{\text {th }}$ ed. W.B. Saunders 1996.

[14] Gotlow J, Laurell L, Rylander H, et al. Treatment of infrabony defect in monkey with bioresorbable and nonresorbable guided tissue regeneration devices. J Dental Res 1993; 721.

[15] Polson A, Garrett S, Stoller N, Greenstein G, Polson AP, Harrold C, Laster L. Guided tissue regeneration in human furcation defects after using a biodegradable barrier: a multicenter feasibility study. J Periodontol 1995; 66: 377. http://dx.doi.org/10.1902/jop.1995.66.5.377

[16] Prato GP, Clauser C, Cortellini P, Tinti C, Vincenzi G, Pagliaro U. Guided tissue regeneration versus mucogingival surgery in the treatment of the human buccal recessions. A 4 year follow up study. J Periodontol 1996; 67: 1216.

\section{http://dx.doi.org/10.1902/jop.1996.67.11.1216}

[17] Glossary of Periodontal Terms, 3rd edition. Chicago, II: American Academy of Periodontology 1992.

[18] Ueda M, Yamada Y, Ozawa R, Okazaki Y. Clinical case reports of injectable tissue-engineered bone for alveolar augmentation with simultaneous implant placement. Int $\mathrm{J}$ Periodontics Restorative Dent 2005; 25: 129-37.

[19] Abukawa H, Shin M, Williams WB, Vacanti JP, Kaban LB, Troulis MJ. Reconstruction of mandibular defects with autologous tissue-engineered bone. J Oral Maxillofac Surg 2004; 62: 601-606. http://dx.doi.org/10.1016/j.joms.2003.11.010

[20] Warnke $\mathrm{PH}$, Springer IN, Wiltfang J, Acil $\mathrm{Y}$, Eufinger $\mathrm{H}$, Wehmoller $\mathrm{M}$, et al. Growth and transplantation of a custom vascularised bone graft in a man. Lancet 2004; 364: 766-70. http://dx.doi.org/10.1016/S0140-6736(04)16935-3

[21] Hokugo A, Kubo Y, Takahashi Y, Fukuda A, Horiuchi K, Mushimoto $\mathrm{K}$, et al. Prefabrication of vascularized bone graft using guided bone regeneration. Tissue Eng 2004; 10: 97886. 